\title{
Placoid pigment epitheliopathy with retinal vasculitis and papillitis
}

\author{
T. H. KIRKHAM, *T. J. FFYTGHE, AND M. D. SANDERS \\ From the Department of Neuro-Ophthalmology, the National Hospital, Queen Square, \\ *and Moorfields Eye Hospital, High Holborn, London
}

Attention was drawn by Gass (1968) to a distinctive fundus appearance of numerous, widespread, flat, yellowish-white lesions at the level of the retinal pigment epithelium and choroid, associated with rapid loss of visual function. Gass proposed the descriptive name "acute posterior multifocal placoid pigment epitheliopathy" for this condition and noted the spontaneous resolution of the lesions which occurred after several months with improvement in the visual acuity.

This report concerns two patients in whom the clinical and fluorescein angiographic findings conform to the description given by Gass (1968) but in whom retinal vasculitis and, in one case, papillitis, were additional features.

\section{Case reports}

Case I, a 26-year-old male carpenter, was admitted to the National Hospital (A60882) under the care of Dr. C. J. Earl, complaining of bilateral visual impairment.

He had developed an "inflammation" of the right lower eyelid 2 months before admission; this had resolved spontaneously after I week and shortly after this episode he noticed blurring of the vision in the right eye. He was seen at his local hospital and the visual acuity was found to be $1 / 60$ in the right eye and 6/6 in the left. Bilateral optic disc swelling was detected and the appearances were interpreted as those of an atypical retrobulbar neuritis. Treatment was commenced with 80 units of ACTH daily. He began to experience intermittent flashes of light in both eyes lasting for 2 or 3 seconds at a time which continued irregularly throughout the day, and 2 weeks after the onset of this symptom the visual acuity in the left eye deteriorated to $6 / 18$. There was no relevant past medical or family history.

\section{Examination}

Visual acuity in the right eye was counting fingers, $\mathrm{N}_{4} 8$, with absent colour vision (Ishihara), and in the left eye $6 / 18, N_{5}$, with normal colour vision. Perimetry showed bilateral central scotomata with a ring scotoma present in the right eye and a broad superior arcuate defect in the left eye (Fig. I, overleaf). The right pupil showed a relative afferent defect. The ocular movements were normal. Slit-lamp examination showed the anterior chamber and vitreous to be clear. The intraocular pressures were normal.

Fundus examination showed multiple, discrete, flat, yellow lesions, some of which were irregularly pigmented and distributed in the postequatorial area. The placoid lesions involved the macula in the right eye but the left macula appeared normal. The optic discs were slightly swollen. The 

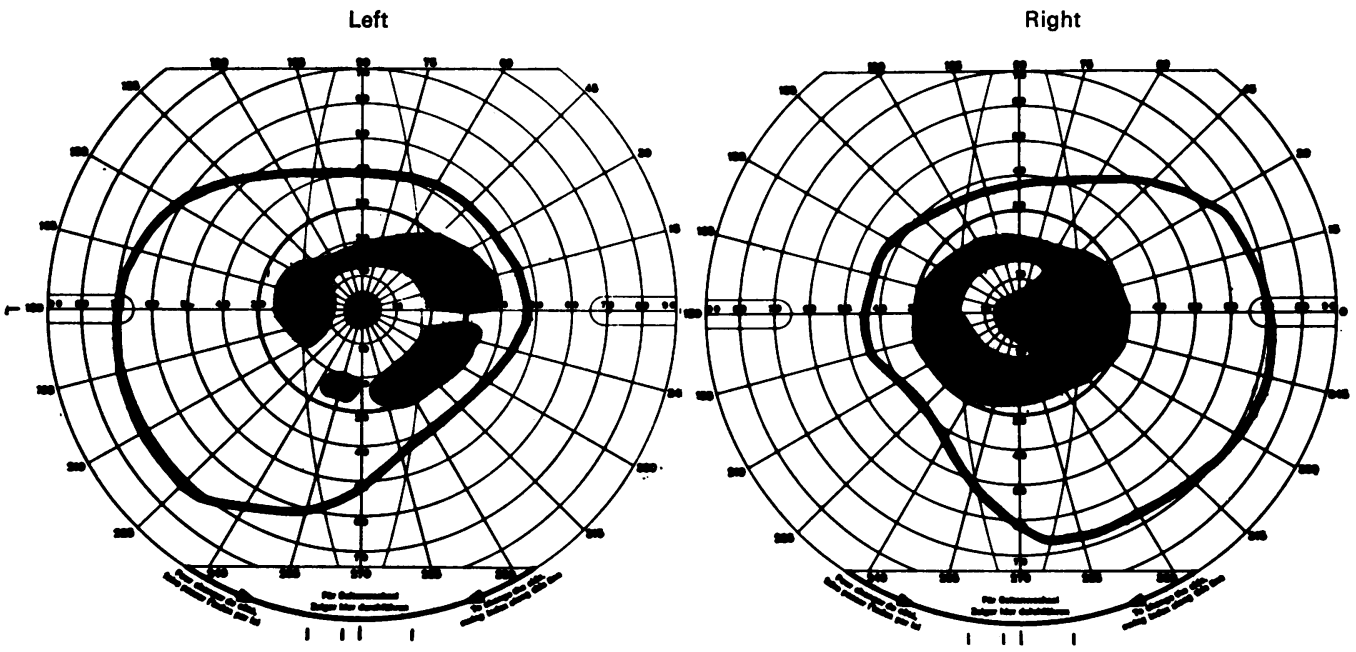

FI G. I Visual fields of Case I when first seen. Goldmann perimeter; white object; size I4

retinal arteries were normal, the veins were distended and irregular in calibre, and there was spontaneous venous pulsation at the optic discs. Small superficial linear haemorrhages were present in the retina, being related to the superior and inferior temporal vessels in both eyes.

\section{Angiography}

Fluorescein angiography was performed using intravenous 20 per cent. sodium fluorescein and the modified Carl Zeiss camera. The arterial phase of the angiogram showed a number of irregular areas of varying size where choroidal fluorescence was completely obscured and which correspond to the placoid areas seen ophthalmoscopically (Figs 2 and 3 ). The non-fluorescent areas gradually began to stain with dye and the 5 -minute photographs showed them to be brightly fluorescent (Fig. 4, opposite).

(2)

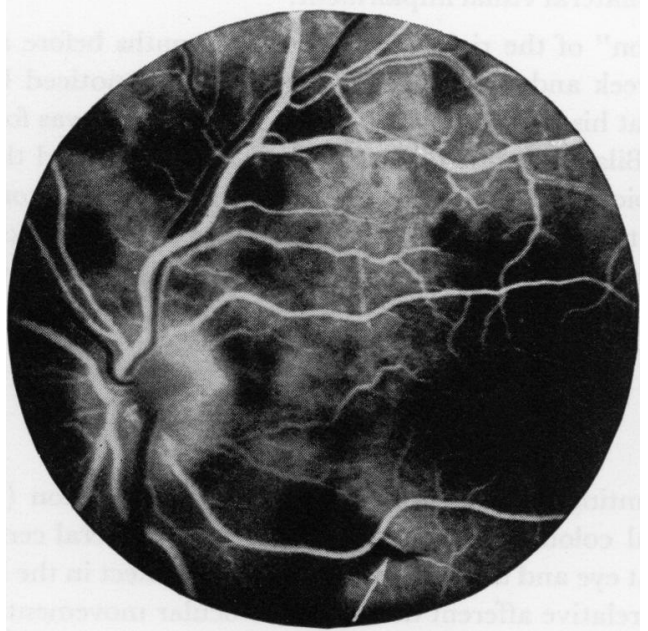

(3)

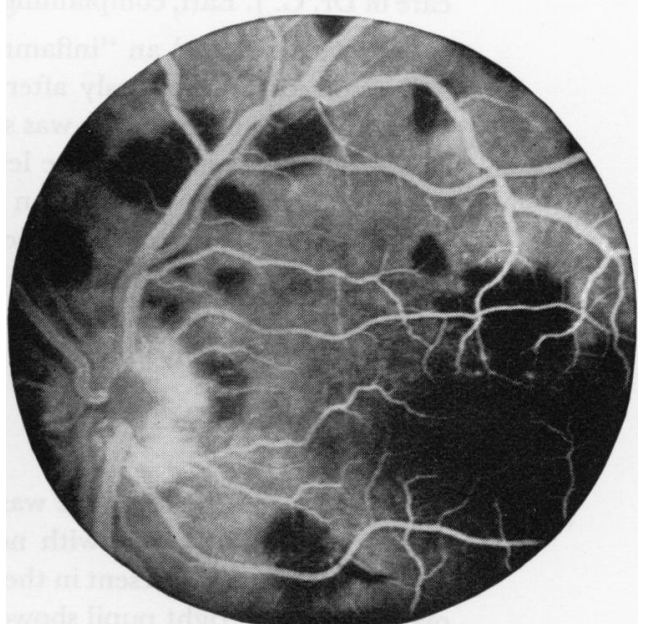

FIG. 2 Case r. Arterio-venous phase of fluorescein angiogram of left eye, showing areas of absent choroidal filling corresponding to the placoid lesions. There is early hyperfluorescence of the optic disc and a superficial retinal haemorrhage (arrow)

FIG. 3 Venous phase, showing clear demarcation of placoid areas. Note irregularity of calibre of upper temporal vein 
The deep plexus of vessels on the disc was markedly dilated; dye began to leak from these vessels in the early stages and there was marked leakage of dye from the disc involving the surrounding retina at I5 minutes (Fig. 5). The rate of flow through the retinal vascular system was of normal velocity and the arteries appeared healthy. The retinal veins showed irregularly distributed focal areas of dilatation, particularly in the superior and inferior temporal branches, and progressive staining of the vessel walls with dye occurred (Fig. 4) with leakage of dye into the surrounding retina. The areas of venous leakage were unrelated to arterio-venous crossings or to the underlying areas of pigmentary disturbance.
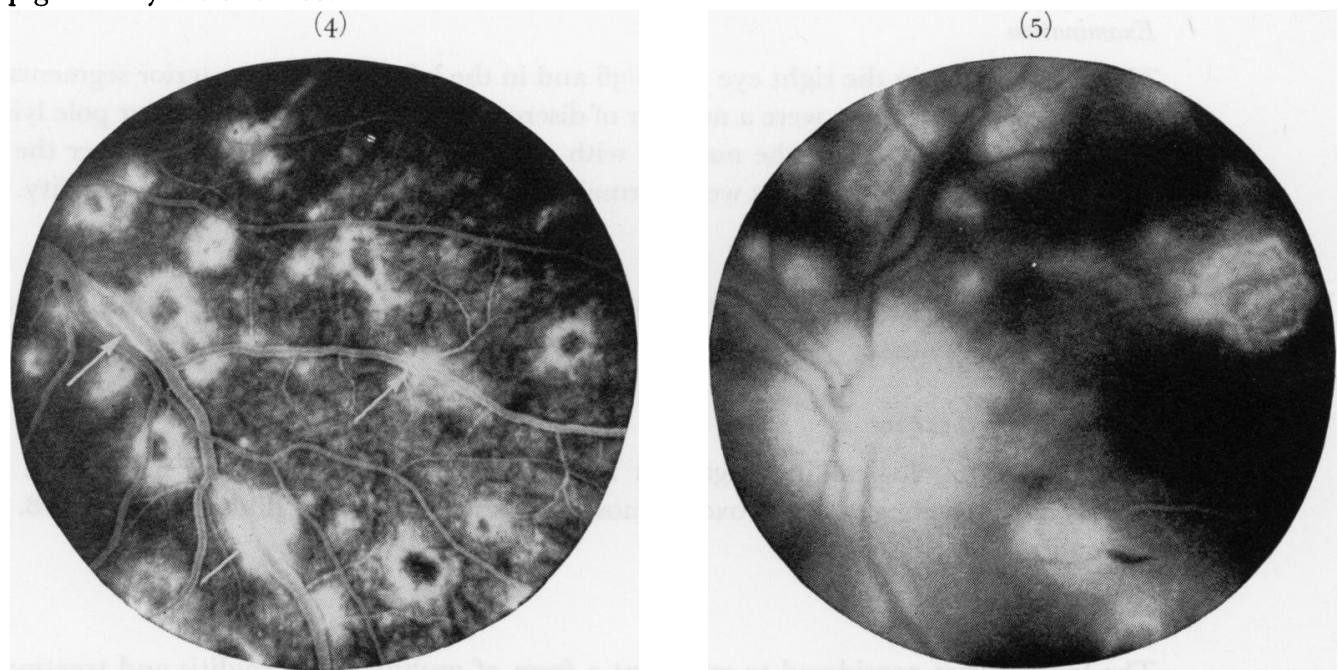

FIG. 4 Infero-temporal region of left eye, showing peripheral hyperfluorescence of placoid lesions, and three areas (arrows) of focal leakage from retinal veins

FIG. 5 Residual appearance, I5 minutes after injection. The placoid areas stain completely with fuorescein, and the hyperfluorescence of the disc extends into the surrounding retina

\section{Laboratory investigations}

Haemoglobin 13.5 g./100 ml.; white blood count 8,80o/cu.mm. (normal differential); erythrocyte sedimentation rate $10 \mathrm{~mm}$./1st hr. Plasma urea, electrocytes, calcium, phosphate, alkaline phosphatase, and proteins normal. Wassermann reaction negative. Paul-Bunnell test, monospot slide test, and sheep cell screening test negative.

Lumbar puncture showed a normal opening pressure.

Cerebrospinal fluid; protein $30 \mathrm{mg}$./ $100 \mathrm{ml}$., lymphocytes 2/cu.mm., Wassermann reaction negative.

Urine examination normal.

Chest, skull, and optic foramina were $x$-rayed and appeared normal. Electroencephalogram normal. Brain scan normal. Electromyogram of deltoid and brachio-radialis muscles normal.

\section{Treatment}

The ACTH injections were discontinued and the visual acuity slowly improved.

\section{Progress}

When the patient was seen 3 months after the onset of symptoms the visual acuity in the right eye was $6 / 36, \mathrm{~N} 8$, and half the Ishihara plates were appreciated; in the left it was $6 / 6, \mathrm{~N}_{5}$, with normal colour vision. Perimetry showed the presence of multiple small scotomatous defects.

There was some resolution of the placoid lesions which remained as pale areas and insome of which there were now more marked pigmentary changes. Needle-shaped superficial retinal haemorrhages were present in the right eye but no haemorrhages were seen in the left retina. 
Fluorescein angiography was repeated and showed that some of the placoid lesions had disappeared and that most of them were reduced in size. There were fewer foci of leakage of dye from the retinal veins and less leakage from the optic disc than at the previous examination.

Case 2, 31-year-old female domestic worker, attended the Westminster Hospital (3322 I8) under the care of Mr. P. D. Trevor-Roper complaining of deterioration of vision in the right eye for 2 weeks. There was no history of recent illness or of exposure to drugs.

Examination

The visual acuity in the right eye was $6 / 36$ and in the left $6 / 6$. The anterior segments were normal. In the right fundus there were a number of discrete white areas at the posterior pole lying deep to the retina particularly around the macula, with minimal elevation of the retina over the lesions. The optic disc and peripheral retina were normal. The left fundus showed no abnormality.

\section{Angiography}

Fluorescein studies showed that the abnormal areas failed to fill initially with dye but later showed marked fluorescence.

\section{Laboratory investigations}

Routine haematological investigations normal. Wassermann reaction and Kahn test negative. Paul-Bunnell test negative. Toxoplasmosis dye test positive to a dilution of $\mathrm{I}$ in 128 . Chest $x$-ray normal.

\section{Treatment}

The lesions were considered to represent a form of multifocal choroiditis and treatment with pred- 8 nisolone $20 \mathrm{mg}$. daily was commenced. Over the next 5 months the visual acuity in the right eye improved to $6 / 12$ and the dosage of prednisolone was gradually reduced.

\section{Progress}

6 months after the original episode, the patient complained that the visual acuity in the left eye had begun to deteriorate. The acuity in the right eye was 6/12 and in the left 6/24. Examination of the right fundus showed that the subretinal swellings previously noted had subsided. In the macular area there were patches of pigment epithelial degeneration and scarring, and these changes extended into the peripheral retina. The left fundus showed several circumscribed whitish areas surrounding the macula with elevation of the overlying retina in places and horizontal folds in the internal limiting membrane. In one area in the infero-temporal region there was evidence of a local phlebitis.

Fluorescein studies showed that in the right eye the picture has altered considerably. The multifocal pigment epithelial changes had formed discrete patches extending into the periphery giving rise to a mosaic appearance. The lesions in the left eye did not fluoresce initially except at their margins but 40 minutes after injection there was definite staining which produced a geographical pattern. The inferior temporal vein showed a segment of focal leakage of dye into the surrounding retina (Fig. 6).

In view of the deterioration in the left eye the dosage of prednisolone was increased and a retrobulbar injection of triamcinolone was given.

After 2 months the visual acuity in the right eye was $6 / 24$ but that in the left had deteriorated to counting fingers. Haemoglobin, white cell count, and erythrocyte sedimentation rate were again found to be normal. The toxoplasmosis dye test was positive to a dilution of $\mathrm{I}$ in 256 .

A year after the onset of the condition the visual acuity was 6/12 in the right eye and counting fingers in the left. The fundi showed bilateral pigment epithelial scarring with deposits of pigment scattering throughout the fundus and resolution of focal pale areas. In the left eye several linear 


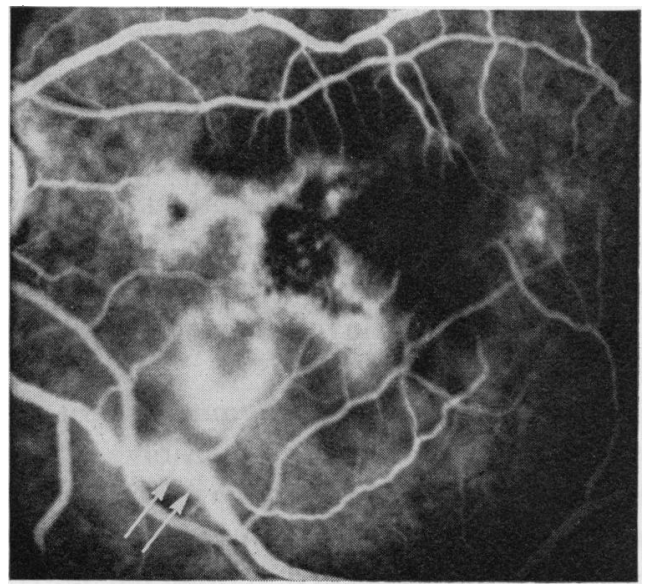

FIG. 6 Case 2. Late arterio-venous phase of fluorescein angiogram of left eye, showing hyperfluorescence of placoid areas and focal area of fluorescein leakage in relation to a retinal vein (arrows)

cracks in Bruch's membrane were also present but the retinal folds had disappeared. The fluorescein studies now showed a diffuse mosaic pigment epithelial disturbance, not only affecting the posterior pole but extending into the periphery. The area of vasculitis previously noted in the left eye had now subsided. It was thought that the condition was unlikely to be influenced further by steroids and these were therefore discontinued.

\section{Discussion}

Placoid epitheliopathy is rarely described but presents an extremely distinctive fundus picture and it seems likely that patients with this condition have previously been considered to have disseminated choroiditis. An interesting report by Neubert (1955) of a patient with sarcoidosis was accompanied by a fundus painting which closely resembled the lesions of placoid epitheliopathy and which the author himself thought to differ from his concept of old, inactive, disseminated choroiditis.

The fundus appearances and fluorescein angiographic studies in the two patients reported above conform to the description of placoid epitheliopathy (Gass, 1968, I970; Van Buskirk, Lessell, and Friedman, I97 I), but the presence of retinal vascular disease and of papillitis has not been previously described. The superficial haemorrhages in the retina were unrelated to the areas of venous endothelial incompetence demonstrated by the fluorescein angiographic study and presumably reflect concomitant retinal microvascular disease. The areas of focal retinal vasculitis did not correspond to the areas of underlying pigment disturbance, although in some areas they coincided.

Fluorescein angiography showed areas of choroidal non-fluorescence which were irregular in shape and of varying size and differed from the triangular-shaped segments of irregular choroidal filling seen in the early phases of a normal fluorescein angiogram. The non-fluorescent areas presumably represent either non-filling of the choriocapillaris or swelling of the overlying pigment and may reflect choroidal vascular disease. The gradual fluorescence of the lesions, which is most marked at their periphery, probably indicates slow diffusion of dye into the lesions and perhaps into the overlying retinal pigment epithelium. Gass (1970) believes that, in the acute phase, the pigment epithelium has cloudy cytoplasm and altered pigment which obscure the normal choroidal fluorescence and that dye later diffuses into the affected cells. In the normal subject fluorescein is not transmitted across Bruch's membrane (Grayson and Laties, I97I) and the integrity of this metabolic barrier may be defective in placoid epitheliopathy. 
Visible macular involvement by placoid lesions has been assumed to be responsible for the central loss in previously reported cases and in our Case 2, but it seems possible that unrecognized concomitant optic nerve involvement by vascular disease may be partly responsible. The visual field defect in the case of Van Buskirk and others (197I) closely resembles that seen in our Case $\mathrm{I}$. The mild swelling of the optic discs, the impairment of visual acuity, and the presence of a relative right afferent pupillary defect and of central and arcuate scotomata in Case I caused initial diagnostic confusion. The presence of optic neuritis in placoid epitheliopathy probably reflects involvement by the disease process of the choroidal blood supply to the optic nerve head.

Gass (1968, 1970) interpreted the clinical and fluorescein angiographic appearances as representing an acute cellular response to an unknown agent, possibly viral, by the pigment epithelium or the adjacent choroid. Van Buskirk and others (197I) thought that the lesions represented a focal choroidal vasculopathy rather than a primary pigment epitheliopathy. We believe that the presence of the associated retinal haemorrhages and the fluorescein angiographic demonstration of segmental retinal vasculitis in our patients support the interpretation that placoid epitheliopathy represents a choroidal vasculitis and that retinal vasculitis may be an occasional additional feature.

No systemic disease was associated with the ocular condition described by Gass (1968), although two of his three patients had positive tuberculin skin tests. The girl with placoid epitheliopathy reported by Van Buskirk and others (1971) developed erythema nodosum after an attack of streptococcal pharyngitis which was treated with penicillin.

Local hypersensitivity on the part of the choroidal and retinal vascular endothelium to an unknown stimulus may be the explanation for the clinical features of placoid epitheliopathy. The rapid onset of symptoms in our Case I after an eyelid infection, the possibly significant increase in the toxoplasmosis dye titre in Case 2, and the features of hypersensitivity demonstrated in previous case reports may be evidence in support of this concept. All reported cases, including the present patients, have been treated with corticosteroids, and it is possible that this in some way modified the natural course of the lesions, although the role of steroids in effecting eventual resolution of the lesion must remain speculative.

\section{Summary}

The clinical and fluorescein angiographic findings in two patients with acute posterior multifocal placoid pigment epitheliopathy are described. The additional features present in these patients were retinal vasculitis and papillitis, and it is suggested that this supports the aetiological concept of a vascular disturbance.

We thank Dr. C. J. Earl and Mr. P. D. Trevor-Roper for permission to publish reports on their patients, the Medical Research Council and the Royal National Institute for the Blind for financial support and apparatus, Miss Sue Ford for the fluorescein angiograms, and Miss Josephine Lace for secretarial assistance.

\section{References}

GASs, J. D. M. (1968) Arch. Ophthal. (Chicago), 80, 177.

- (1970) "Stereoscopic Atlas of Macular Disease. A Funduscopic and Angiographic Presentation", p. 128. Mosby, St. Louis

Grayson, M. C., and LATies, A. M. (197I) Arch. Ophthal. (Chicago), 85, 600

NEUBERT, F. R. (1955) Trans. ophthal. Soc. U. K., 75, 200

VAN BUSKIRK, E. M., LesSell, S., and friedman, E. (197I) Arch. Ophthal. (Chicago), 85, 369 